\title{
ANALYTIC APPROXIMATION OF THE DEBYE FUNCTION
}

\author{
K. V. KHISHCHENKO ${ }^{1,2,3^{*}}$ \\ ${ }^{1}$ Joint Institute for High Temperatures of the Russian Academy of Sciences \\ Izhorskaya 13 Bldg 2, 125412 Moscow, Russia \\ ${ }^{2}$ Moscow Institute of Physics and Technology \\ Institutskiy Pereulok 9, 141701 Dolgoprudny, Moscow Region, Russia \\ ${ }^{3}$ South Ural State University \\ Lenin Avenue 76, 454080 Chelyabinsk, Russia \\ *Corresponding author. E-mail: konst@ihed.ras.ru
}

\section{DOI: 10.20948/mathmontis-2020-49-8}

Summary. An expression in a closed form is proposed for the approximation of the Debye function used in thermodynamic models of solids. This expression defines an analytic function that has the same limiting behavior as the Debye function at low and high temperatures. The approximation gives the maximum relative deviation from the value of the Debye function less than 0.001 . The proposed expression can be useful in the equations of state of solids in a wide temperature range.

\section{INTRODUCTION}

The Debye model [1] was proposed for description of thermodynamic behavior of materials in a wide range of temperatures. It represents the phonon contribution to equations of state of solids as an interpolation between limiting cases of low and high temperatures [2-25]. Equations of state of matter are necessary for analysis and numerical simulation of physical phenomena under extreme conditions of high temperatures and high pressures [19,26-40].

Analytic expressions of thermodynamic potentials within the Debye model contain the Debye function in a form of integral [1],

$$
D(x)=\frac{3}{x^{3}} \int_{0}^{x} \frac{t^{3} \mathrm{~d} t}{\mathrm{e}^{t}-1},
$$

$x>0$, which cannot be expressed in elementary functions. Despite of that this integral can be written as analytic expression with infinite series [1,3,41,42] or special functions (polylogarithms and the Riemann zeta function) [43], closed-form expressions approximating the Debye function are interesting for practical use in thermodynamic calculations. Many works are devoted to elaboration of simple approximations of the Debye functions with different ac- 
curacy $[14,44-50]$. All of such approximations can be sorted as piecewise continuously differentiable functions $[44,45,47]$ and smooth (in particular, analytic) functions [14, 46, 48-50].

In the present work, an expression is proposed approximating the Debye function in a closed form of analytic function. Some results of calculations are presented illustrating the accuracy of this approximation.

\section{MODEL OF THERMODYNAMIC PROPERTIES OF SOLIDS}

Thermodynamic potential the Helmholtz free energy is traditionally taken as a basis of equation of state model. This potential can be presented as a sum of three parts:

$$
F(V, T)=F_{\mathrm{c}}(V)+F_{\mathrm{a}}(V, T)+F_{\mathrm{e}}(V, T)
$$

those are a portion of energy corresponding to zero temperature $T=0\left(F_{\mathrm{c}}\right)$ and thermal contributions of ions and electrons ( $F_{\mathrm{a}}$ and $F_{\mathrm{e}}$, respectively). Here $V$ is the specific volume; $T$ is the temperature. Considering thermal contribution of ions in solids, one can take into account portions of energy of acoustical $\left(F_{\mathrm{ac}}\right)$ and optical $\left(F_{\mathrm{o} \alpha}\right)$ modes of ions vibrations. For the unit cell of the crystal structure with $v$ particles, this contribution is as follows:

$$
F_{\mathrm{a}}(V, T)=F_{\mathrm{ac}}(V, T)+\sum_{\alpha=1}^{3(v-1)} F_{\mathrm{o} \alpha}(V, T) .
$$

The energy of acoustic vibration modes is usually considered within the framework of the Debye model [1]:

$$
F_{\mathrm{ac}}(V, T)=\frac{R T}{v}\left[3 \ln \left[1-\exp \left(-\theta_{\mathrm{ac}} / T\right)\right]-D\left(\theta_{\mathrm{ac}} / T\right)\right] .
$$

Optical mode contributions are commonly considered in terms of the Einstein model [51]:

$$
F_{\mathrm{o} \alpha}(V, T)=\frac{R T}{v} \ln \left[1-\exp \left(-\theta_{\mathrm{ac}} / T\right)\right] .
$$

Here, $\theta_{\mathrm{ac}}$ and $\theta_{\mathrm{o} \alpha}$ are the characteristic temperatures of the acoustical and optical modes of ions vibrations.

The first and second derivatives of the Helmholtz free energy with respect to temperature determine the entropy and isochoric heat capacity of a substance:

$$
\begin{gathered}
S=-(\partial F / \partial T)_{V} \\
C_{V}=T(\partial S / \partial T)_{V} .
\end{gathered}
$$


Consequently, the first and second derivatives of the Debye function appear in the entropy and the isochoric heat capacity of solids. In particular, if the characteristic temperature $\theta_{\mathrm{ac}}$ does not depend on temperature, one can obtain the following expressions for the contributions of acoustic modes:

$$
\begin{gathered}
S_{\mathrm{ac}}=-\frac{R}{v}\left[3 \ln [1-\exp (-x)]-\frac{3 x}{\mathrm{e}^{x}-1}-D(x)+x D^{\prime}(x)\right], \\
C_{V \mathrm{ac}}=\frac{R}{v}\left[\frac{3 x^{2} \mathrm{e}^{x}}{\left(\mathrm{e}^{x}-1\right)^{2}}+x^{2} D^{\prime \prime}(x)\right],
\end{gathered}
$$

where $x=\theta_{\mathrm{ac}} / T$.

Sometimes, it is convenient to take into account the properties of the Debye function:

$$
\begin{gathered}
D^{\prime}(x)=\frac{3}{\mathrm{e}^{x}-1}-\frac{3}{x} D(x) \\
D^{\prime \prime}(x)=-\frac{3 \mathrm{e}^{x}}{\left(\mathrm{e}^{x}-1\right)^{2}}-\frac{3}{x}\left[\frac{3}{\mathrm{e}^{x}-1}-\frac{4}{x} D(x)\right] .
\end{gathered}
$$

Then the entropy and specific heat capacity are related to the value of the Debye function:

$$
\begin{gathered}
S_{\mathrm{ac}}=-\frac{R}{v}[3 \ln [1-\exp (-x)]-4 D(x)], \\
C_{V \mathrm{ac}}=\frac{3 R}{v}\left[4 D(x)-\frac{3 x}{\mathrm{e}^{x}-1}\right] .
\end{gathered}
$$

However, relations (10) and (11) may not be valid for approximation functions used instead of the Debye function. Then using equations (12) and (13) will lead to some inaccuracy.

\section{INFINITE-SERIES FORMS OF THE DEBYE FUNCTION}

Following Debye [1], one can rewrite integral in equation (1) and obtain

$$
D(x)=\frac{3}{x^{3}} \int_{0}^{\infty} \frac{t^{3} \mathrm{~d} t}{\mathrm{e}^{t}-1}-\frac{3}{x^{3}} \int_{x}^{\infty} \frac{t^{3} \mathrm{~d} t}{\mathrm{e}^{t}-1} .
$$

The first integral in equation (14) has known value $\pi^{4} / 15$ [1,52]; the last integral in equation (14) can be simplified using the Taylor series

$$
\frac{1}{1-y}=\sum_{k=0}^{\infty} y^{k}
$$


which is convergent for $|y|<1$, and integrated by parts:

$$
\int_{x}^{\infty} \frac{t^{3} \mathrm{~d} t}{\mathrm{e}^{t}-1}=\int_{x}^{\infty} t^{3} \mathrm{e}^{-t} \sum_{k=0}^{\infty} \mathrm{e}^{-k t} \mathrm{~d} t=\sum_{k=1}^{\infty} \int_{x}^{\infty} t^{3} \mathrm{e}^{-k t} \mathrm{~d} t=\sum_{k=1}^{\infty}\left(\frac{x^{3}}{k}+\frac{3 x^{2}}{k^{2}}+\frac{6 x}{k^{3}}+\frac{6}{k^{4}}\right) \mathrm{e}^{-k t} .
$$

So, one obtains

$$
D(x)=\frac{\pi^{4}}{5 x^{3}}-3 \sum_{k=1}^{\infty} \frac{1}{k}\left(1+\frac{3}{k x}+\frac{6}{k^{2} x^{2}}+\frac{6}{k^{3} x^{3}}\right) \mathrm{e}^{-k t}
$$

for $x>0$.

At high temperatures, one can use the following relation [53]:

$$
\frac{t}{\mathrm{e}^{t}-1}=\sum_{n=0}^{\infty} \frac{B_{n}}{n !} t^{n}
$$

which is convergent for $|t|<2 \pi$. Here, $B_{n}$ are the Bernoulli numbers [53]. One obtains

$$
\int_{0}^{x} \frac{t^{3} \mathrm{~d} t}{\mathrm{e}^{t}-1}=\int_{0}^{x} t^{2} \sum_{n=0}^{\infty} \frac{B_{n}}{n !} t^{n} \mathrm{~d} t=\sum_{n=0}^{\infty} \frac{B_{n}}{n !} \int_{0}^{x} t^{n+2} \mathrm{~d} t=\sum_{n=0}^{\infty} \frac{B_{n}}{n !} \frac{x^{n+3}}{n+3} .
$$

So,

$$
D(x)=\sum_{n=0}^{\infty} \frac{3 B_{n}}{(n+3) n !} x^{n}
$$

for $|x|<2 \pi$.

\section{APPROXIMATION FORM}

Truncated series (17) is normally used as the basis of approximation of the Debye function at low temperatures.

In this work, a similar form of approximation function is proposed:

$$
K_{L M}(x)=A_{03} x^{-3}-\sum_{l=1}^{L}\left(A_{l 0}+A_{l 1} x^{-1}+A_{l 2} x^{-2}+A_{l 3} x^{-3}\right) \mathrm{e}^{-l x}
$$

Evidently, the value of first-term coefficient

$$
A_{03}=\frac{\pi^{4}}{5}
$$


secures the same limiting behavior of the function $K_{L M}(x)$ and all its derivatives as the Debye function $D(x)$ and its derivatives for $x \rightarrow \infty$.

Using the Taylor series

$$
\mathrm{e}^{y}=\sum_{n=0}^{\infty} \frac{1}{n !} y^{n}
$$

one can evaluate limiting behavior of the function $K_{L M}(x)$ for $x \rightarrow 0$ :

$$
K_{L M}(x)=A_{03} x^{-3}-\sum_{n=0}^{\infty} \sum_{l=1}^{L} \frac{1}{n !}(-l)^{n}\left(A_{l 0} x^{n}+A_{l 1} x^{n-1}+A_{l 2} x^{n-2}+A_{l 3} x^{n-3}\right) .
$$

Representing series (24) in the form

$$
K_{L M}(x)=\sum_{m=-3}^{\infty} C_{m} x^{m}
$$

one can easily obtain sequence of relations between coefficients of equations (24) and (25):

$$
\begin{gathered}
C_{-3}=A_{03}-\sum_{l=1}^{L} A_{l 3}, \\
C_{-2}=-\sum_{l=1}^{L}\left(A_{l 2}-l A_{l 3}\right) \\
C_{-1}=-\frac{1}{2} \sum_{l=1}^{L}\left(2 A_{l 1}-2 l A_{l 2}+l^{2} A_{l 3}\right)
\end{gathered}
$$

and

$$
C_{m}=-\sum_{l=1}^{L} \frac{(-l)^{m}}{(m+3) !}\left((m+1)(m+2)(m+3) A_{l 0}-(m+2)(m+3) l A_{l 1}+(m+3) l^{2} A_{l 2}-l^{3} A_{l 3}\right)
$$

for $m \geqslant 0$.

Comparing form (25) with series (20), one can formulate conditions of coincidence of limit- 
ing behavior of the function $K_{L M}(x)$ and its derivatives up to order $M \leqslant 4(L-1)$ for $x \rightarrow 0$ :

$$
\begin{gathered}
A_{03}-\sum_{l=1}^{L} A_{l 3}=0, \\
-\sum_{l=1}^{L}\left(A_{l 2}-l A_{l 3}\right)=0, \\
-\sum_{l=1}^{L}\left(2 A_{l 1}-2 l A_{l 2}+l^{2} A_{l 3}\right)=0
\end{gathered}
$$

and

$$
\begin{gathered}
-\sum_{l=1}^{L}(-l)^{m}\left((m+1)(m+2)(m+3) A_{l 0}-(m+2)(m+3) l A_{l 1}+(m+3) l^{2} A_{l 2}-l^{3} A_{l 3}\right) \\
=3(m+1)(m+2) B_{m}
\end{gathered}
$$

for $0 \leqslant m \leqslant M$.

Solving this system of $M+4$ equations, one obtains $M+4$ coefficients $\left(A_{L 3}, A_{L 2}, A_{L 1}, A_{L 0}\right.$, $A_{(L-1) 3}$ and so on, if any) of the function $K_{L M}(x)$ (21) with the same limiting behavior as the Debye function $D(x)$ for $x \rightarrow 0$ up to order $M$ of derivatives.

Values of the rest of coefficients $A_{10}, A_{11}, A_{12}, A_{13}, A_{20}$ and so on (if any) can be naturally taken from series (17):

$$
A_{l 0}=\frac{3}{l}, A_{l 1}=\frac{9}{l^{2}}, A_{l 2}=\frac{18}{l^{3}}, A_{l 3}=\frac{18}{l^{4}} .
$$

\section{APPROXIMATION WITH L $=1$}

For $L=1$, the approximation function $K_{L M}(x)$ has the only variant $K_{10}(x)$ with 4 coefficients $A_{l i}$ with $l=1, i=0,1,2$ and 3 :

$$
A_{10}=\frac{\pi^{4}}{30}-1, A_{11}=\frac{\pi^{4}}{10}, A_{12}=\frac{\pi^{4}}{5}, A_{13}=\frac{\pi^{4}}{5} .
$$

Calculated values of the function $K_{10}(x)$ and its first and second derivatives $K_{10}^{\prime}(x)$ and $K_{10}^{\prime \prime}(x)$ are shown in figures 1-3 in comparison with the values of the Debye function $D(x)$ and its derivatives $D^{\prime}(x)$ and $D^{\prime \prime}(x)$. In addition, the relative deviations of the function $K_{10}(x)$ and its derivatives $K_{10}^{\prime}(x)$ and $K_{10}^{\prime \prime}(x)$ from the reference function $D(x)$ and its derivatives $D^{\prime}(x)$ and $D^{\prime \prime}(x)$ are presented in figures $1(b), 2(b)$ and $3(b)$, respectively. The reference values of $D(x)$, $D^{\prime}(x)$ and $D^{\prime \prime}(x)$ were calculated using truncated series (17) with $k \leqslant 12$ for $x>3.34$ and (20) with $n \leqslant 66$ for $x \leqslant 3.34$. 


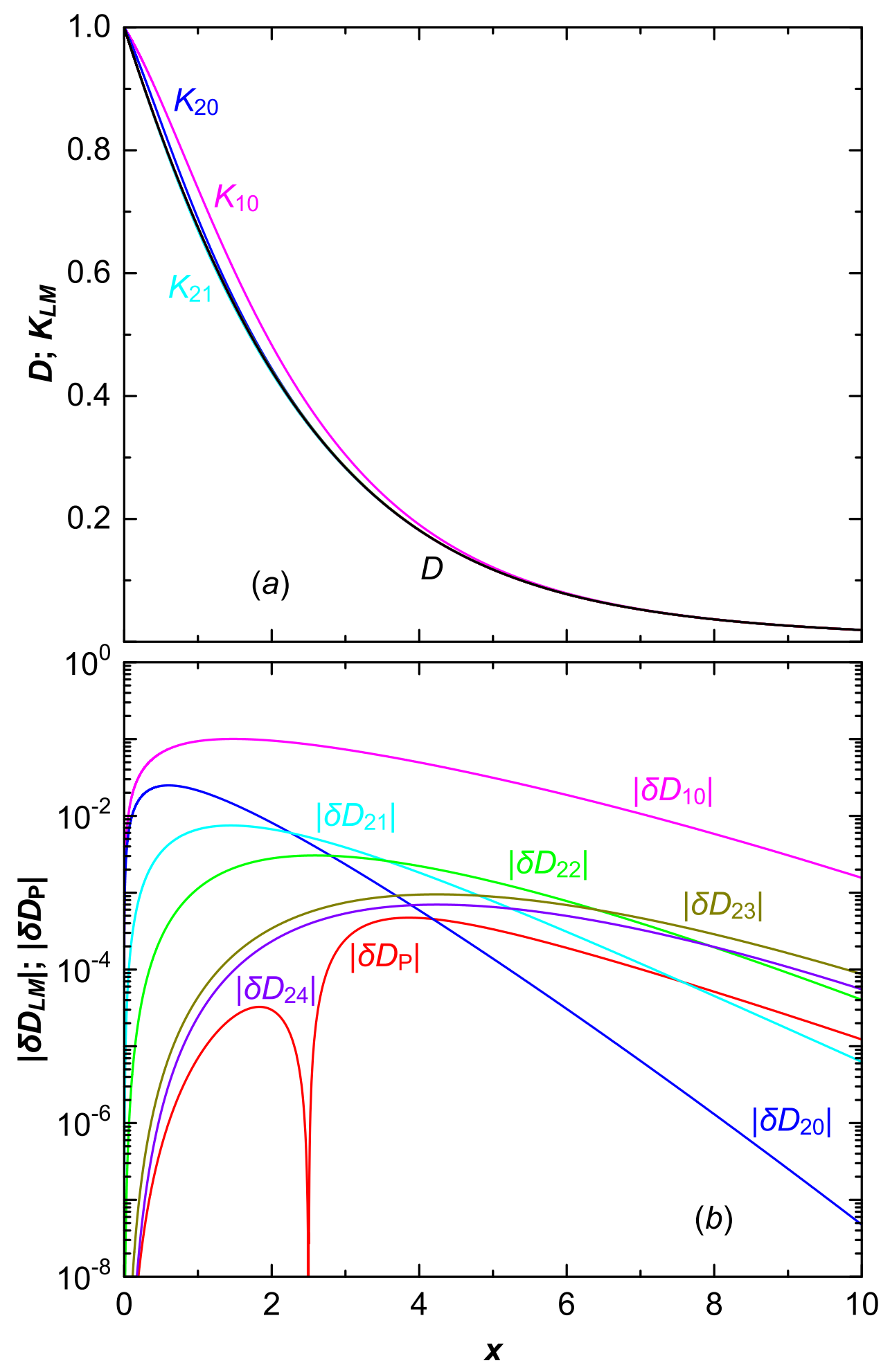

Figure 1: (a) The Debye function $D(x)$ and the approximation functions $K_{L M}(x)$. (b) The absolute values of the relative deviations of the functions $K_{L M}(x)$ from the reference function $D(x),\left|\delta D_{L M}(x)\right|=\left|1-K_{L M}(x) / D(x)\right|$; $\left|\delta D_{\mathrm{P}}(x)\right|=\left|1-D_{\mathrm{P}}(x) / D(x)\right|$, where $D_{\mathrm{P}}(x)$ is the approximation function by Prut [47]. 


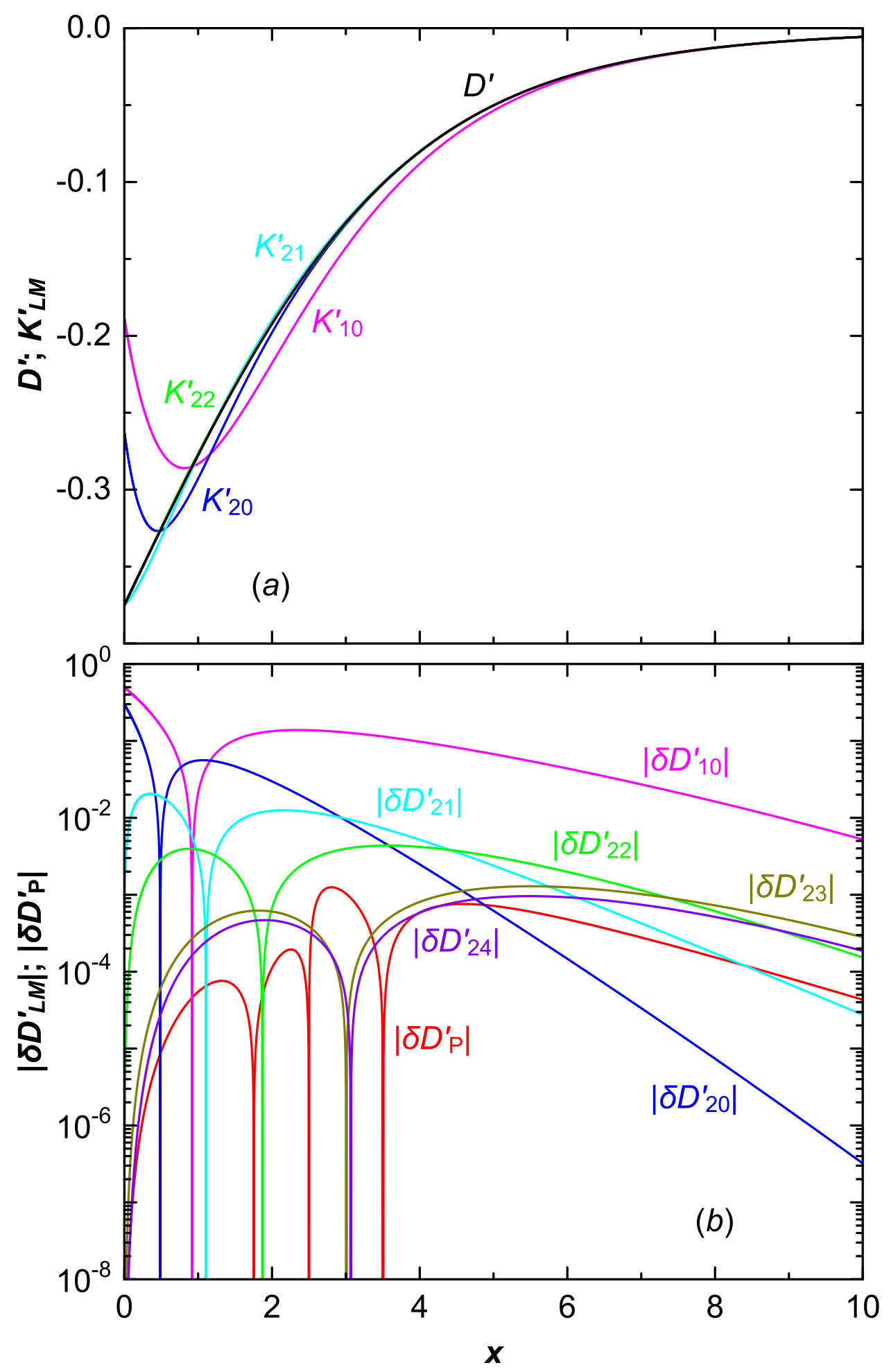

Figure 2: (a) The first derivative with respect to $x$ of the Debye function, $D^{\prime}(x)$, and the approximation functions, $K_{L M}^{\prime}(x)$. (b) The absolute values of the relative deviations of the derivatives $K_{L M}^{\prime}(x)$ and $D_{\mathrm{P}}^{\prime}(x)$ from the reference derivative $D^{\prime}(x),\left|\delta D_{L M}^{\prime}(x)\right|=\left|1-K_{L M}^{\prime}(x) / D^{\prime}(x)\right|$ and $\left|\delta D_{\mathrm{P}}^{\prime}(x)\right|=\left|1-D_{\mathrm{P}}^{\prime}(x) / D^{\prime}(x)\right|$. 


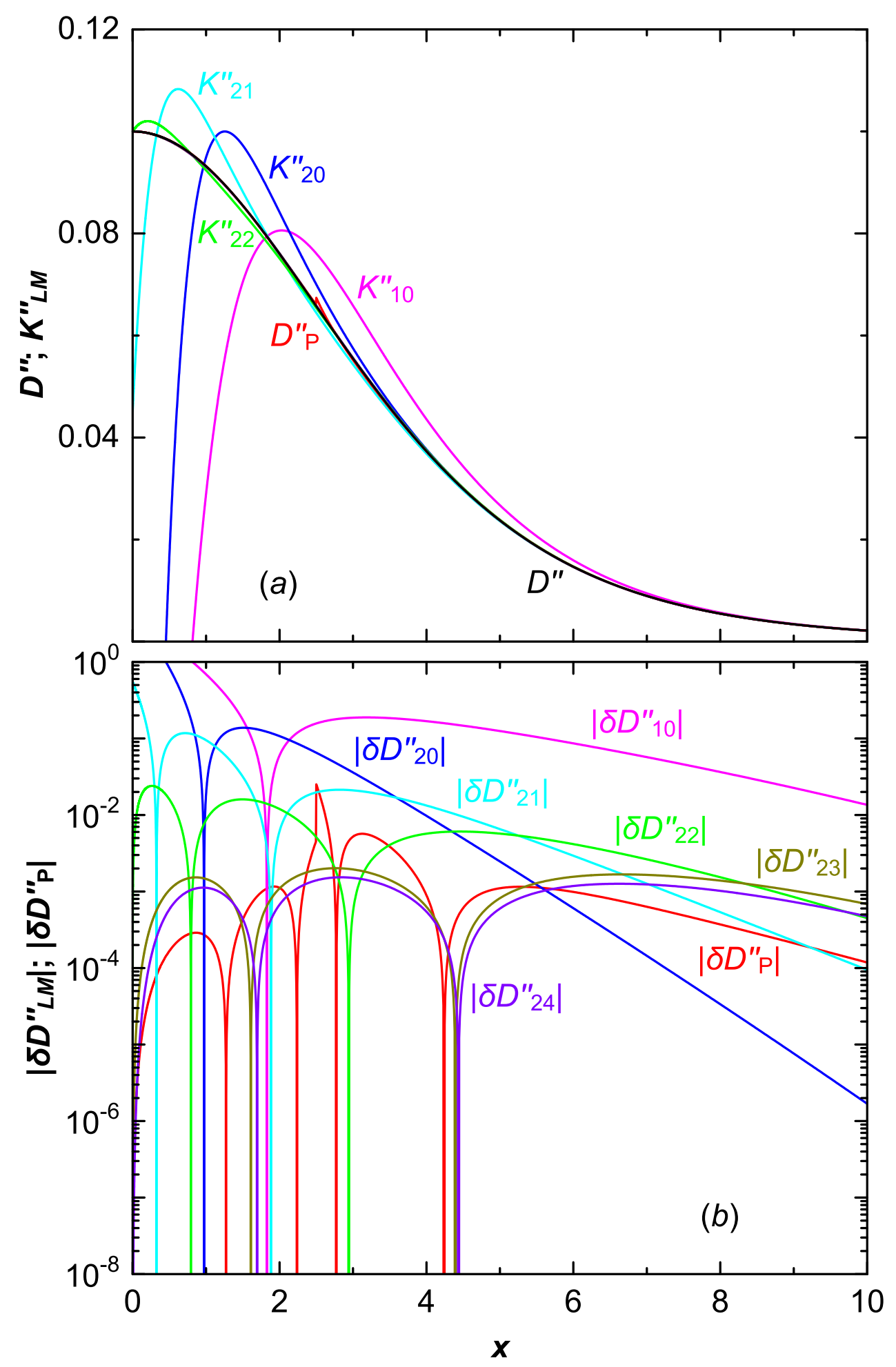

Figure 3: (a) The second derivative with respect to $x$ of the Debye function, $D^{\prime \prime}(x)$, and the approximation functions, $K_{L M}^{\prime \prime}(x)$ and $D_{\mathrm{P}}^{\prime \prime}(x)$. (b) The absolute values of the relative deviations of the derivatives $K_{L M}^{\prime \prime}(x)$ and $D_{\mathrm{P}}^{\prime \prime}(x)$ from the reference derivative $D^{\prime \prime}(x),\left|\delta D_{L M}^{\prime \prime}(x)\right|=\left|1-K_{L M}^{\prime \prime}(x) / D^{\prime \prime}(x)\right|$ and $\left|\delta D_{\mathrm{P}}^{\prime \prime}(x)\right|=\left|1-D_{\mathrm{P}}^{\prime \prime}(x) / D^{\prime \prime}(x)\right|$. 
The maximum absolute values of the relative deviations in the region $x>0$ are approximately 0.1 for $K_{10}(x), 0.5$ for $K_{10}^{\prime}(x)$ and 4.0 for $K_{10}^{\prime \prime}(x)$. Note that the relative deviation of the third derivative $K_{10}^{\prime \prime \prime}(x)$ from the reference $D^{\prime \prime \prime}(x)$ grows at $x \rightarrow 0$ in inverse proportion to $x$.

The ratios of the isochoric heat capacity $C_{V}(x)$ to its value in the high-temperature limit $(x \rightarrow 0), C_{\mathrm{h}}=3 R / v$, from equation (9) with the approximation derivative $K_{10}^{\prime \prime}(x)$ and from equation (13) with the approximation function $K_{10}(x)$ are shown in figure 4 in comparison with the reference dependence $C_{V}(x) / C_{\mathrm{h}}$ that is obtained using equation (9) with the reference $D^{\prime \prime}(x)$.

The maximum absolute value of the relative deviation from the reference dependence $C_{V}(x)$ is approximately 0.07 for the case of equation (9) with $K_{10}^{\prime \prime}(x)$ and 0.3 for the case of equation (13) with $K_{10}(x)$, as one can see in figure $4(b)$.

\section{APPROXIMATIONS WITH L $=2$}

For $L=2$, the approximation function $K_{L M}(x)$ has 5 variants $K_{2 M}(x)$ for $M=0,1,2,3$ and 4 with 8 coefficients $A_{l i}$ at $l=1$ and $2, i=0,1,2$ and 3:

$$
\begin{gathered}
\left.A_{10}=3 \text { (for } M=0,1,2 \text { and } 3\right) \text { or } A_{10}=\frac{8 \pi^{4}}{15}-49(\text { for } M=4) \\
A_{11}=9(\text { for } M=0,1 \text { and } 2) \text { or } A_{11}=\frac{8 \pi^{4}}{5}-\frac{219}{2}-12 A_{10}(\text { for } M=3 \text { and } 4) \\
A_{12}=18(\text { for } M=0 \text { and } 1) \text { or } A_{12}=\frac{16 \pi^{4}}{5}-117-36 A_{10}-8 A_{11}(\text { for } M=2,3 \text { and } 4), \\
A_{13}=18(\text { for } M=0) \text { or } A_{13}=\frac{16 \pi^{4}}{5}-39-24 A_{10}-12 A_{11}-4 A_{12}(\text { for } M=1,2,3 \text { and } 4), \\
A_{20}=\frac{4 \pi^{4}}{15}-1-A_{10}-A_{11}-\frac{1}{2} A_{12}-\frac{1}{6} A_{13} \\
A_{21}=\frac{2 \pi^{4}}{5}-A_{11}-A_{12}-\frac{1}{2} A_{13} \\
A_{22}=\frac{2 \pi^{4}}{5}-A_{12}-A_{13} \\
A_{23}=\frac{\pi^{4}}{5}-A_{13}
\end{gathered}
$$

Calculated values of the functions $K_{2 M}(x)$ and their first and second derivatives $K_{2 M}^{\prime}(x)$ and $K_{2 M}^{\prime \prime}(x)$ are shown in figures $1-3$. One can see that the functions $K_{20}(x)$ and $K_{21}(x)$, as well as the function $K_{10}(x)$, are easily distinguishable from the reference function $D(x)$ in figure $1(a)$.

The derivatives $K_{2 M}^{\prime}(x)$ and $K_{2 M}^{\prime \prime}(x)$ with $M=0,1$ and 2, as well as the derivatives $K_{10}^{\prime}(x)$ and $K_{10}^{\prime \prime}(x)$, are also easily distinguishable from the reference derivatives $D^{\prime}(x)$ and $D^{\prime \prime}(x)$ in figures 2(a) and 3(a). 


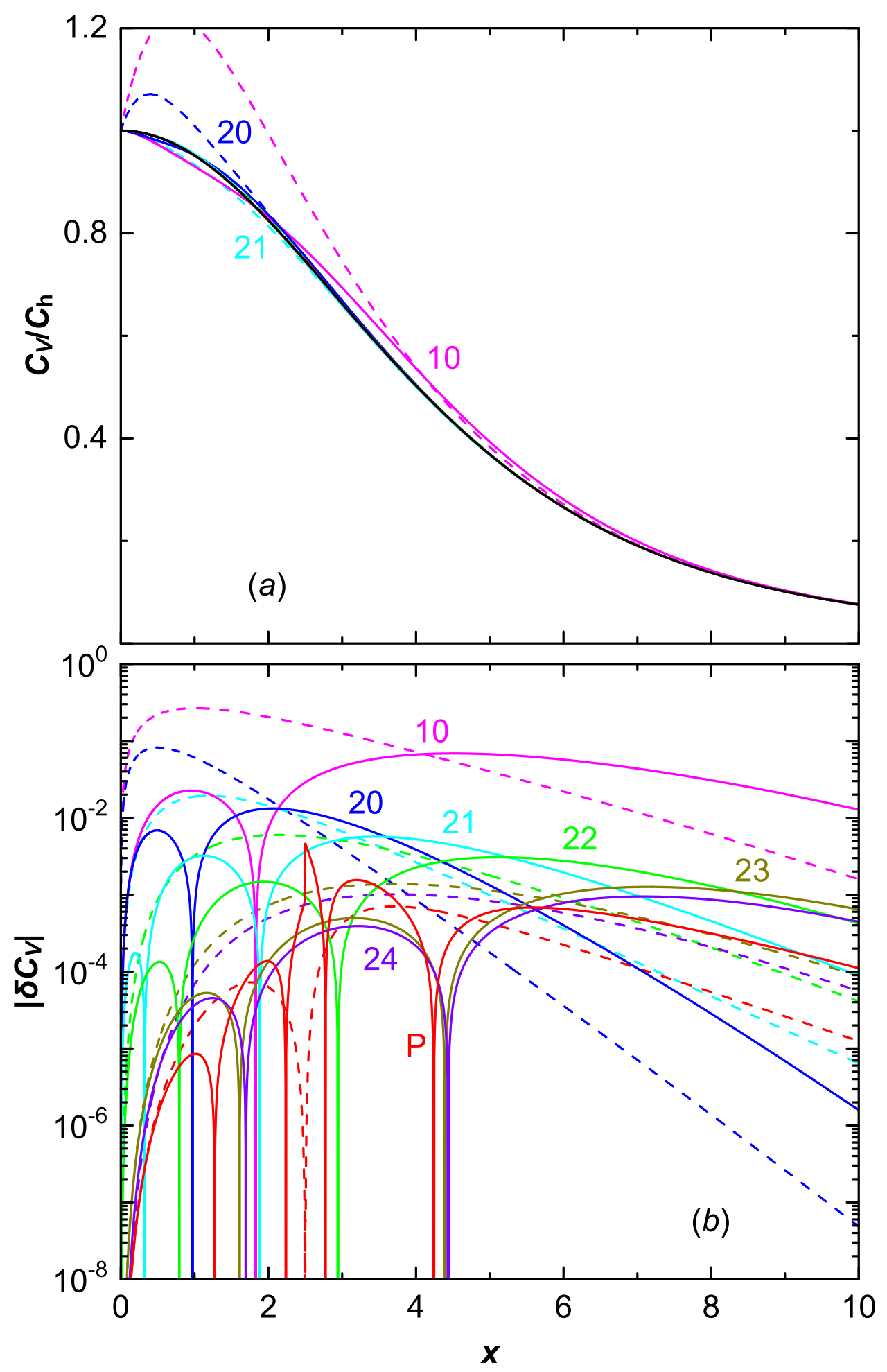

Figure 4: (a) The reference (black line) and approximation ratios $C_{V}(x) / C_{\mathrm{h}}$ (colored lines) and $(b)$ the absolute values of the relative deviations $\left|\delta C_{V \mathrm{~A}}(x)\right|$ of the approximation dependences $(\mathrm{A}=L M$ and $\mathrm{P})$ from the reference $C_{V}(x)$ : solid lines-equation (9) with $K_{L M}^{\prime \prime}(x)$ and $D_{\mathrm{P}}^{\prime \prime}(x)$; dashed lines-equation (13) with $K_{L M}(x)$ and $D_{\mathrm{P}}(x)$. 


\begin{tabular}{ccccccc}
\hline $\mathrm{A}$ & $\left|\delta D_{\mathrm{A}}\right| \mathrm{m}$ & $\left|\delta D_{\mathrm{A}}^{\prime}\right|_{\mathrm{m}}$ & $\left|\delta D_{\mathrm{A}}^{\prime \prime}\right|_{\mathrm{m}}$ & $\left|\delta D_{\mathrm{A}}^{\prime \prime \prime}\right|_{\mathrm{m}}$ & $\left|\delta C_{V \mathrm{~A}(9)}\right|_{\mathrm{m}}$ & $\left|\delta C_{V \mathrm{~A}(13)}\right| \mathrm{m}$ \\
\hline 10 & $1.00 \times 10^{-1}$ & $4.98 \times 10^{-1}$ & $3.99 \times 10^{+0}$ & $\infty$ & $6.87 \times 10^{-2}$ & $2.66 \times 10^{-1}$ \\
20 & $2.49 \times 10^{-2}$ & $3.01 \times 10^{-1}$ & $4.61 \times 10^{+0}$ & $\infty$ & $1.32 \times 10^{-2}$ & $8.20 \times 10^{-2}$ \\
21 & $7.50 \times 10^{-3}$ & $2.05 \times 10^{-2}$ & $5.48 \times 10^{-1}$ & $\infty$ & $5.68 \times 10^{-3}$ & $1.94 \times 10^{-2}$ \\
22 & $3.05 \times 10^{-3}$ & $4.36 \times 10^{-3}$ & $2.40 \times 10^{-2}$ & $\infty$ & $3.07 \times 10^{-3}$ & $6.00 \times 10^{-3}$ \\
23 & $9.51 \times 10^{-4}$ & $1.29 \times 10^{-3}$ & $2.02 \times 10^{-3}$ & $9.70 \times 10^{-2}$ & $1.27 \times 10^{-3}$ & $1.38 \times 10^{-3}$ \\
24 & $6.99 \times 10^{-4}$ & $9.59 \times 10^{-4}$ & $1.53 \times 10^{-3}$ & $3.69 \times 10^{-2}$ & $9.48 \times 10^{-4}$ & $1.01 \times 10^{-3}$ \\
$\mathrm{P}$ & $4.73 \times 10^{-4}$ & $1.25 \times 10^{-3}$ & $2.52 \times 10^{-2}$ & $5.42 \times 10^{-1}$ & $4.62 \times 10^{-3}$ & $7.06 \times 10^{-4}$ \\
\hline
\end{tabular}

Table 1: Maximum absolute values of the relative deviations $\delta D_{\mathrm{A}}(x), \delta D_{\mathrm{A}}^{\prime}(x), \delta D_{\mathrm{A}}^{\prime \prime}(x), \delta D_{\mathrm{A}}^{\prime \prime \prime}(x)$ and $\delta C_{V \mathrm{~A}}(x)$ for $x>0$, where the cases $\mathrm{A}=L M$ and $\mathrm{P}$ correspond to the approximation functions $K_{L M}(21)$ and $D_{\mathrm{P}}$ [47]; the last two columns correspond to the use of equations (9) and

(13), respectively.

The remaining functions $K_{2 M}(x)$ and derivatives $K_{2 M}^{\prime}(x)$ and $K_{2 M}^{\prime \prime}(x)$ almost coincide with the reference dependences $D(x), D^{\prime}(x)$ and $D^{\prime \prime}(x)$ in figures $1(a), 2(a)$ and $3(a)$.

The absolute values of the relative deviations of the function $K_{2 M}(x)$ and their derivatives $K_{2 M}^{\prime}(x)$ and $K_{2 M}^{\prime \prime}(x)$ from the reference function $D(x)$ and its derivatives $D^{\prime}(x)$ and $D^{\prime \prime}(x)$ are presented in figures $1(b), 2(b)$ and $3(b)$, respectively. One can see that the maxima of these absolute values for $L=2$ are less than the corresponding maxima for $K_{10}(x), K_{10}^{\prime}(x)$ and $K_{10}^{\prime \prime}(x)$. These maxima at $L=2$ decrease monotonically with increasing $M$ (table 1).

In the best case for $L=2$, the maximum absolute values of the relative deviations for $x>0$ are approximately 0.0007 for $K_{24}(x), 0.001$ for $K_{24}^{\prime}(x), 0.002$ for $K_{24}^{\prime \prime}(x)$ and 0.04 for $K_{24}^{\prime \prime \prime}(x)$.

Note that, for $L=2$ and $M=0,1$ and 2, as well as for $L=1$, the relative deviations of the third derivatives $K_{2 M}^{\prime \prime \prime}(x)$ from the reference derivative $D^{\prime \prime \prime}(x)$ grow at $x \rightarrow 0$ in inverse proportion to $x$.

The ratios of the isochoric heat capacity $C_{V}(x) / C_{\mathrm{h}}$ from equation (9) with the approximation derivatives $K_{2 M}^{\prime \prime}(x)$ and from equation (13) with the approximation functions $K_{2 M}(x)$ are shown in figure 4. One can see that, for $L=2$ and $M=0$ and 1 , as well as for $L=1$, these ratios are easily distinguishable from the reference dependence $C_{V}(x) / C_{\mathrm{h}}$ in figure $4(a)$. For $L=2$ and $M=2$, the dependence $C_{V}(x) / C_{\mathrm{h}}$ can be distinguished in the case of the use of equation (13) with the approximation function $K_{22}(x)$. The dependences $C_{V}(x) / C_{\mathrm{h}}$ for the remaining cases of $L=2$ and $M=2,3$ and 4 almost coincide with the corresponding reference dependence in figure 4(a).

As one can see in figure 4(b) and table 1, the maximum absolute values of the relative deviations $\left|\delta C_{V L M}\right|_{\mathrm{m}}$ (for $x>0$ ) decrease monotonically with increasing $L$ from 1 to 2 and with 
increasing $M$ from 0 to 4 . Moreover, using equation (13) gives a higher relative deviation than using equation (9). In the best case for $L=2$ and $M=4$, the maximum absolute value of the relative deviation $\left|\delta C_{V L M}\right|_{\mathrm{m}}$ in the region $x>0$ is approximately 0.0009 using equation (9).

For comparison, the results of using the piecewise continuously differentiable approximation function $D_{\mathrm{P}}(x)$ [47] are presented in figures 1-4 and table 1. Unlike analytic functions $K_{L M}(x)$, using equation (13) with $D_{\mathrm{P}}(x)$ gives a lower relative deviation than using equation (9) with discontinuous derivative $D_{\mathrm{P}}^{\prime \prime}(x)$. Despite the slightly lower values of the maximum deviations $\left|\delta D_{\mathrm{P}}\right|_{\mathrm{m}} \approx 0.0005$ and $\left|\delta C_{V \mathrm{P}(13)}\right|_{\mathrm{m}} \approx 0.0007$, the use of the analytic approximation function $K_{24}(x)$ seems preferable in thermodynamic models.

\section{CONCLUSIONS}

Thus, a family of analytic functions $K_{L M}(x)$ is proposed that approximates the Debye function $D(x), x>0$, in closed form. Among these functions with $L=1$ and 2, the case of $K_{24}(x)$ for $x>0$ gives the lowest maximum relative deviations of the function and its first and second derivatives from the reference function $D(x)$ (less than 0.0007) and its derivatives $D^{\prime}(x)$ (less than 0.001 ) and $D^{\prime \prime}(x)$ (less than 0.002), as well as the lowest maximum relative deviation for the value of the isochoric heat capacity (less than 0.001 ). The proposed expressions can be useful in modeling the equations of state for solids in a wide range of temperatures and densities.

Acknowledgments: The present work is financially supported by the Russian Foundation for Basic Research (grant No. 18-08-01493).

The paper is based on the proceedings of the XXXVI International Conference on Interaction of Intense Energy Fluxes with Matter, Elbrus, the Kabardino-Balkar Republic of the Russian Federation, March 1 to 6, 2021.

\section{REFERENCES}

[1] P. Debye, "Zur Theorie der spezifischen Wärmen", Ann. Phys., 344(14), 789-839 (1912).

[2] L. V. Al'tshuler, "Use of shock waves in high-pressure physics", Sov. Phys. Usp., 8(1), 52-91 (1965).

[3] V. N.Zharkov and V. A. Kalinin, Equations of State for Solids at High Pressures and Temperatures, Boston, MA: Springer, (1971).

[4] L. D. Landau and E. M. Lifshitz, Statistical Physics. Part 1, Course of Theoretical Physics, Vol. 5, Oxford: Pergamon, (1980).

[5] A. V. Bushman and V.E. Fortov, "Models of equation of the matter state", Usp. Fiz. Nauk, 140(2), 177-232 (1983).

[6] G. I. Kerley, "Theoretical equation of state for aluminum", Int. J. Impact Eng., 5(1-4), 441-449 (1987).

[7] V. S. Vorobev, "Potential interpolation of Debye approximation for the description of liquid and gas phases", Zh. Eksp. Teor. Fiz., 109(1), 162-173 (1996).

[8] K. V. Khishchenko, I. V. Lomonosov, V. E. Fortov, and O. F. Shlenskii, "Thermodynamical properties of plastics in wide range of densities and temperatures", Dokl. Akad. Nauk, 349(3), 322-325 (1996). 
[9] K. V. Khishchenko, "Temperature and heat capacity of polymethyl methacrylate behind the front of strong shock waves", High Temp., 35(6), 991-994 (1997).

[10] W. B. Holzapfel, "Equations of state for solids under strong compression", Z. Kristallogr., 216(9), 473-488 (2001).

[11] L. F. Gudarenko, M. V.Zhernokletov, S. I. Kirshanov, A. E. Kovalev, V. G. Kudel'kin, T. Lebedeva, A. I. Lomaikin, M. A. Mochalov, G. V. Simakov, A. N. Shuikin, and I. M. Voskoboinikov, "Properties of shock-compressed Carbogal. Equations of state for Carbogal and Plexiglas", Combust., Explos. Shock Waves, 40(3), 344-355 (2004).

[12] K. V. Khishchenko, V.E. Fortov, and I. V.Lomonosov, "Multiphase equation of state for carbon over wide range of temperatures and pressures", Int. J. Thermophys., 26(2), 479-491 (2005).

[13] E. Eser, H. Koc, B. A. Mamedov, and I. M. Askerov, "Estimation of the heat capacity of some semiconductor compounds using $n$-dimensional Debye functions", Int. J. Thermophys., 32(10), 2163 2169 (2011).

[14] R. J. Goetsch, V. K. Anand, A. Pandey, and D. C. Johnston, "Structural, thermal, magnetic, and electronic transport properties of the $\mathrm{LaNi}_{2}\left(\mathrm{Ge}_{1-x} \mathrm{P}_{x}\right)_{2}$ system", Phys. Rev. B, 85(5), 054517 (2012).

[15] I. Roslyakova, B. Sundman, H. Dette, L. Zhang, and I. Steinbach, "Modeling of Gibbs energies of pure elements down to $0 \mathrm{~K}$ using segmented regression", CALPHAD: Comput. Coupling Phase Diagrams Thermochem., 55(2), 165-180 (2016).

[16] H. Liu, H. Song, Q. Zhang, G. Zhang, and Y. Zhao, "Validation for equation of state in wide regime: Copper as prototype", Matter Radiat. Extremes, 1(2), 123-131 (2016).

[17] E. Yakub, "The modified Debye-Grüneisen model for highly compressed diamond", J. Low Temp. Phys., 187(1-2), 20-32 (2017).

[18] O. A. Lukianova and V. V. Sirota, "Dielectric properties of silicon nitride ceramics produced by free sintering", Ceram. Int., 43(11), 8284-8288 (2017).

[19] I. V. Lomonosov and S. V. Fortova, "Wide-range semiempirical equations of state of matter for numerical simulation on high-energy processes", High Temp., 55(4), 585-610 (2017).

[20] Q. Wang, T. Li, H. Wang, H. Li, Y. Miao, Q. Chen, M. Wan, L. Chen, J. Sun, K. He, and G. Zheng, "The thermodynamic, electronic and optical properties of GeP type $\mathrm{ZnO}$ under pressure calculated by Debye model and hybrid function", Mater. Chem. Phys., 211, 206-213 (2018).

[21] A. V. Khvan, A. T. Dinsdale, I. A. Uspenskaya, M. Zhilin, T. Babkina, and A. M. Phiri, "A thermodynamic description of data for pure $\mathrm{Pb}$ from $0 \mathrm{~K}$ using the expanded Einstein model for the solid and the two state model for the liquid phase", CALPHAD: Comput. Coupling Phase Diagrams Thermochem., 60, 144-155 (2018).

[22] X.Zhang, G. Wang, B.Luo, F. Tan, S.N.Bland, J.Zhao, C. Sun, and C. Liu, "Refractive index and polarizability of polystyrene under shock compression", J. Mater. Sci., 53(17), 12628-12640 (2018).

[23] S. Sh. Rekhviashvili, A. A. Sokurov, and M. M. Bukhurova, "Heat capacity of an ordered bundle of single-walled carbon nanotubes", High Temp., 57(4), 482-485 (2019).

[24] S. D. Gilev, "Low-parametric equation of state of aluminum", High Temp., 58(2), 166-172 (2020).

[25] R. Tomaschitz, "Effective partition function of crystals: Reconstruction from heat capacity data and Debye-Waller factor", Phys. B, 593, 412243 (2020).

[26] V. E. Fortov, V. V. Kim, I. V. Lomonosov, A. V. Matveichev, and A. V. Ostrik, "Numerical modeling of hypervelocity impacts", Int. J. Impact Eng., 33(1-12), 244-253 (2006).

[27] M.E. Povarnitsyn, K. V. Khishchenko, and P. R. Levashov, "Hypervelocity impact modeling with different equations of state", Int. J. Impact Eng., 33(1-12), 625-633 (2006).

[28] S. I. Tkachenko, P. R. Levashov, and K. V. Khishchenko, "The influence of an equation of state on the interpretation of electrical conductivity measurements in strongly coupled tungsten plasma", $J$. Phys. A: Math. Gen., 39(23), 7597-7603 (2006).

[29] I. K. Krasyuk, A. Yu. Semenov, I. A. Stuchebryukhov, and K. V. Khishchenko, "Experimental verification of the ablation pressure dependence upon the laser intensity at pulsed irradiation of metals", J. Phys.: Conf. Ser., 774, 012110 (2016).

[30] K. V. Khishchenko, "Equation of state of sodium for modeling of shock-wave processes at high pressures", Math. Montis., 40, 140-147 (2017). 
[31] K. K. Maevskii and S. A. Kinelovskii, "Thermodynamic parameters of mixtures with silicon nitride under shock-wave impact in terms of equilibrium model", High Temp., 56(6), 853-858 (2018).

[32] K. K. Maevskii, "Thermodynamic parameters of mixtures with silicon nitride under shock-wave loading", Math. Montis., 45, 52-59 (2019).

[33] M. Hallajisany, J.Zamani, M. S. Salehi, and J. A. Vitoria, "A new model for the time delay between elastic and plastic wave fronts for shock waves propagating in solids", Shock Waves, 29(3), 451-469 (2019).

[34] M. Hallajisany, J.Zamani, and J. A. Vitoria, "Numerical and theoretical determination of various materials Hugoniot relations based on the equation of state in high-temperature shock loading", High Pressure Res., 39(4), 666-690 (2019).

[35] K. V. Khishchenko, "Equation of state for niobium at high pressures", Math. Montis., 47, 119-123 (2020).

[36] S. V. Rykov, V. A. Rykov, I. V. Kudryavtseva, E. E. Ustyuzhanin, and A. V. Sverdlov, "Fundamental equation of state of argon, satisfying the scaling hypothesis and working in the region of high temperatures and pressures", Math. Montis., 47, 124-136 (2020).

[37] V. Yu. Bodryakov, "Isolation of the magnetic contribution to the thermal expansion of nickel at ferromagnetic transformation on the base of analysis of $\beta\left(C_{P}\right)$ correlation dependence", High Temp., 58(2), 213-217 (2020).

[38] T. S. Sokolova, A. I. Seredkina, and P. I. Dorogokupets, "Density patterns of the upper mantle under Asia and the Arctic: Comparison of thermodynamic modelling and geophysical data", Pure Appl. Geophys., 177(9), 4289-4307 (2020).

[39] V.E. Borisov, O. B. Feodoritova, N. D. Novikova, Yu. G. Rykov, and V. T. Zhukov, "Computational model for high-speed multicomponent flows", Math. Montis., 48, 32-42 (2020).

[40] K. V. Khishchenko and A. E. Mayer, "High- and low-entropy layers in solids behind shock and ramp compression waves", Int. J. Mech. Sci., 189, 105971 (2021).

[41] I. I. Guseinov and B. A. Mamedov, "Calculation of integer and noninteger $n$-dimensional Debye functions using binomial coefficients and incomplete gamma functions", Int. J. Thermophys., 28(4), 1420-1426 (2007).

[42] R. Pässler, "Unprecedented integral-free Debye temperature formulas: Sample applications to heat capacities of ZnSe and ZnTe", Adv. Condens. Matter Phys., 2017, 9321439 (2017).

[43] A.E. Dubinov and A. A. Dubinova, "Exact integral-free expressions for the integral Debye functions", Tech. Phys. Lett., 34(12), 999-1001 (2008).

[44] H. C. Thacher Jr, "Rational approximations for the Debye functions", J. Chem. Phys., 32(2), 638 (1960).

[45] T. W. Listerman and C. B. Ross, "A simple method for calculating Debye heat capacity values using the Einstein heat capacity formula", Cryogenics, 19(9), 547-549 (1979).

[46] S. A. Labutin and M. V. Pugin, "Approksimatsionnyye formuly dlya funktsii Debaya", Izv. Vyssh. Uchebn. Zaved., Fiz., 39(2), 103-104 (1996).

[47] V. V. Prut, "Semiempirical model of equation of state for condensed media", High Temp., 43(5), $713-726(2005)$.

[48] N. A. Masyukov and A. V. Dmitriev, "Approximation formulas in the Debye theory of the lowtemperature specific heat of solids", Bull. Russ. Acad. Sci.: Phys., 71(8), 1076-1078 (2007).

[49] W. W. Anderson, "An analytic expression approximating the Debye heat capacity function", AIP $A d v ., 9(7), 075108$ (2019).

[50] M. Bouafia and H. Sadat, "An approximate model for the heat capacity of solids", Int. J. Thermophys., 41(6), 84 (2020).

[51] A. Einstein, "Die Plancksche Theorie der Strahlung und die Theorie der spezifischen Wärme", Ann. Phys., 327(1), 180-190 (1907).

[52] B. D. Sukheeja, "Solution of the integral in Debye's theory of specific heat of solids", Am. J. Phys., 38(7), 923-924 (1970).

[53] D. E. Knuth and T. J. Buckholtz, "Computation of tangent, Euler, and Bernoulli numbers", Math. Comput., 21(100), 663-688 (1967). 